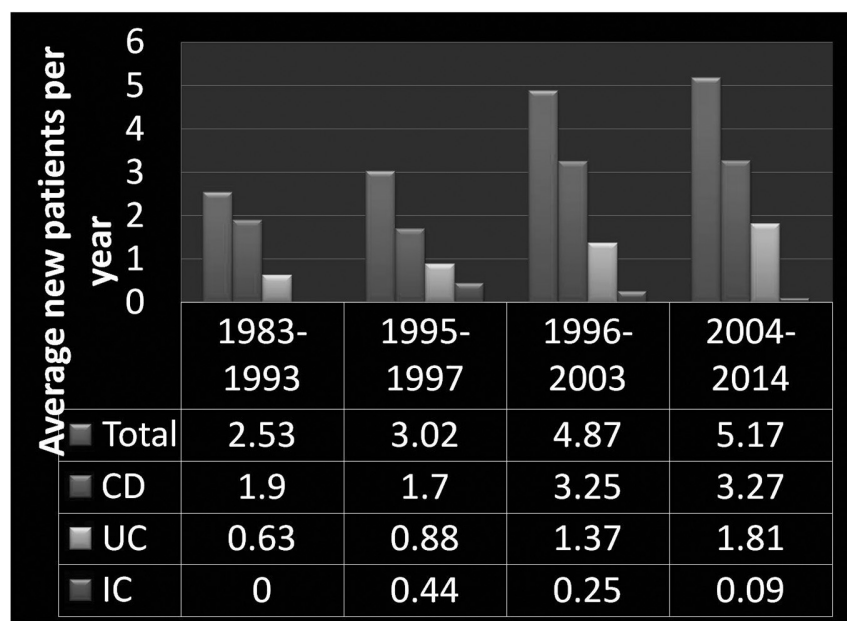

Abstract G353 Figure 1

Introduction The incidence of paediatric inflammatory bowel disease (IBD) has risen significantly across Europe in the last 20 years, although our own data had suggested that this had plateaued by 2004 . We have now studied the data from the same area over the last decade and compared them with our previous studies (1983-2003).

Methods All cases of IBD $<16$ years of age residing in a defined location within our region were prospectively recorded from January 2004 to March 2014. The incidence, age, gender and disease type were analysed and compared to our data from 1983-2003 from the same region.

Results See Table 1. Between 2004 and 2014, there were 57 new patients compared to 39 (1996-2003) and 28 (1983-1993). The overall incidence of IBD was 5.9 per 100,000 per year, Crohn's disease (CD) 3.7 per 100,000 per year and Ulcerative colitis (UC) 2.07 per 100,000 per year compared with 5.4 per 100,000 per year for 1996 to 2003 . There was no statistically significant difference between the two time periods ( $\mathrm{p}$ value of 0.675 ). The median age at diagnosis remains at 12 years with a male to female ratioof 1.7:1.

Conclusion The incidence of paediatric IBD in a defined geographical area within our region has remained similar for more than 15 years with a slight increase in the incidence of UC, suggesting that the previous exponential rise in incidence has reached a stable state.

\section{G354 THE ONGOING RAPID AND SIGNIFICANT RISE OF INCIDENT PAEDIATRIC-ONSET INFLAMMATORY BOWEL DISEASE IN SCOTLAND}

${ }^{1} \mathrm{FA}$ Jagger, ${ }^{2} \mathrm{FL}$ Cameron, ${ }^{3} \mathrm{P}$ Henderson, ${ }^{2} \mathrm{P}$ Rogers, ${ }^{3} \mathrm{P} \mathrm{McGrogan},{ }^{4} \mathrm{~S}$ Loganathan, ${ }^{3}$ RK Russell, ${ }^{3} \mathrm{R}$ Hansen, ${ }^{2} \mathrm{DC}$ Wilson. ${ }^{1}$ University of Aberdeen, Aberdeen, UK; ${ }^{2}$ Child Life and Health, University of Edinburgh, Edinburgh, UK; ${ }^{3}$ Paediatric Gastroenterology, Yorkhill Hospital, Glasgow, UK; ${ }^{4}$ Paediatric Gastroenterology, Royal Aberdeen Children's Hospital, Aberdeen, UK

\subsection{6/archdischild-2015-308599.310}

Background The worldwide incidence of paediatric-onset inflammatory bowel disease (PIBD) is rising, with Scotland having the highest rate in the UK. Scottish PIBD data over the last 40 years has shown a consistent increase, including a $76 \%$ rise over 13 years around the millennium (Henderson P et al. IBD 2012;18:999-1005).
Aims The aim of this study was to calculate current PIBD incidence rates in Scotland and to determine if the temporal trend of significant increase has been maintained.

Methods Historical data from 2003-2008 (cohort 1) was compared to prospective, nationwide data of all incident cases diagnosed in paediatric services (under 16 years of age) from 2009-2013 (cohort 2). Age-sex adjusted incidence rates were calculated using population data from the General Registrar's Office for Scotland. Cases were classified as Crohn's disease (CD), ulcerative colitis (UC) or inflammatory bowel disease unclassified (IBDU) and diagnosed according to the Porto criteria. Statistical analysis was performed using Poisson regression.

Results A total of 436 patients were diagnosed with PIBD over six years in cohort 1 (265 CD, $115 \mathrm{UC}, 56 \mathrm{IBDU})$ compared to 478 children over five years in cohort 2 (286 CD, 126 UC, 66 IBDU). Median age at diagnosis in cohort 2 (60\% males) was 12.3 years, similar to cohort $1(58 \%$ males $)$ at 11.9 years. The adjusted incidence rate increased from 7.8/100,000/year (95\% CI 7.1-8.6) in cohort 1 (2003-2008) to $10.4 / 100,000 /$ year (95\% CI 9.6-11.5) in cohort $2(2009-2013)$ ( $\mathrm{p}<0.001)$. This significant increase was also seen individually for CD (4.7/ 100,000/year [95\% CI 4.2-5.4] compared to 6.3/100,000/year [95\% CI 5.6-7.0] $[\mathrm{p}<0.0001])$ and UC $(2.1 / 100,000 /$ year [95\% CI 1.7-2.5] compared to $2.7 / 100,000 /$ year [95\% CI $2.3-$ 3.3] $[\mathrm{p}=0.009])$. There was a non-significant increase in IBDU from $1.0 / 100,000 /$ year $(95 \%$ CI $0.7,1.3)$ in cohort 1 to $1.4 /$ $100,000 /$ year $(95 \%$ CI $1.1,1.8)$ in cohort 2 ( $p=0.07)$.

Conclusion There continues to be an ongoing rise in incident PIBD (and both CD and UC) in 2009-13 in this national, population-based study compared to recent historical data, with a further significant rise of $33 \%$. The reasons behind this continued increase remain unclear and further research is needed to elucidate potential factors in aetiopathogenesis.

\section{G355 PRESENTING PHENOTYPE OF ULCERATIVE COLITIS (UC) IN CHILDREN 2010-13}

${ }^{1} \mathrm{JJ}$ Ashton, ${ }^{1,2} \mathrm{~T}$ Coelho, ${ }^{2} \mathrm{~S}$ Ennis, ${ }^{1} \mathrm{~A}$ Batra, ${ }^{1} \mathrm{NA}$ Afzal, ${ }^{1} \mathrm{RM}$ Beattie. ${ }^{1}$ Paediatric Gastroenterology, University Hospitals Southampton, Southampton, UK; ${ }^{2}$ Human Genomics, University of Southampton, Southampton, UK

\subsection{6/archdischild-2015-308599.311}

Aims There has been a two-fold increase in the incidence of paediatric-onset UC over the last 20 years; there are few recent reports of the presenting phenotype - symptoms, inflammatory markers and disease extent. We report the presenting features of a defined cohort and compare to previous data.

Methods Patients diagnosed with UC at University Hospitals Southampton from 2010-2013 were identified from an in-house database. Data were obtained from note review using a standardised proforma and compared to previous UK data. ${ }^{1}$ Weight and height at diagnosis are presented as median SDS (95\% CI).

Results 49 children were included. Median age 13.18 (Range 1.59-16.24 years), 27 male. The most common presenting features are seen in Table 1. Weight loss (36.7\%) and lethargy $(36.7 \%)$ were less common. The majority of patients presented with pancolitis $(68.1 \%)$.

A significant number of patients present with normal inflammatory markers (percentage with normal CRP 69.4\%, normal ESR 34.9\%). Median CRP $3.0 \mathrm{mg} / \mathrm{L}(0.0-12-9)$, ESR21.5 mm/ hr (15.2-26.8)). 